\section{Application of the Monti channel in female adult with urethral injuries in pelvic trauma}

\author{
Hanchao Zhang, Ashok Kumar Kunwar, \\ Yi Dai, Deyi Luo, Hong Shen \\ Department of Urology, West China \\ Hospital of Sichuan University, Chengdu, \\ Sichuan, China
}

\section{Abstract}

Objective. To report 3 cases using the Monti ileovesicostomy as a continent catheterizable stoma in women with post-traumatic resulted in loss of the urethra.

Materials and Methods. Between April and November 2010, the Monti technique was performed as a reconstructive procedure in 3 female patients at our institution. All cases had history of posterior urethral defect, two motor vehicle accidents, and one pelvic crush injury. Channels were created by transversely reconfigured segment of ileum. The 3 patients age was 20,41 , and 43 years. Indications, technique, complications and outcomes are reported below.

Results. Three patients were undergone for transversely re-tubularized ileal segment urinary reconstruction. All of them were evaluated from 5 to 12 months postoperatively. All patients' postoperative visits to our outpatient gynecological clinic about 3,6 and 12 months respectively after Monti ileovesicostomy operation revealed no dehiscence, necrosis, stenosis or perforation of the tube. All performed catheterization through the conduit without difficulty.

Conclusion. The Monti ileovesicostomy is a new technique for creation of a continent catheterizable stoma. Based on our data, Monti procedure seems to be a valuable technique in patients with complicated urethral defect who cannot be managed with routine urethroplastic techniques. This reconstruction provides another option for a diversion technique in patients with irreparable urethral damage. However, evaluation whether it can be extensively developed still need more investigations with larger number of patients by long-term follow up.

\section{Introduction}

Urethral injuries in women are rare events since the female urethra is short and mobile, without any significant attachments to the pubic bone. They are usually accompanied by severe pelvic fractures, where bony fragments of the fractured pelvis provoke lacerations of the urethra. ${ }^{1}$ Structures and defects of the posterior urethra in female is one of the most significant clinical complications concerning urologist. ${ }^{2}$ Posterior urethral injuries in pelvic fracture were estimated at $5-10 \%$ in previous studies. ${ }^{3}$ Pelvic fracture urethral distraction defect (PFUDD) is usually managed by the end to end anastomotic urethroplasty. However, in some cases the urethral defect is so long or complete damaged, that it cannot be negotiated with vigorous releasing of urethra from surrounding tissue, inferior pubectomy and even re-routing maneuvers. ${ }^{4,5}$ Based on the location and length of the defects, various techniques have been used in such cases including onlay repairs, stricture excision with augmented anastomosis, a tubularized flap of sigmoid colon, and free or vascularized skin flap, etc. Certainly, many complications have also been related to these techniques such as restenosis and necrosis of urethra, and the patients have to tolerate the treatment of urethral dilation in the long run. ${ }^{5,6}$ Other options such as perineostomy or suprapubic tube could also be used as salvage procedure. ${ }^{7,8}$ Nevertheless, it compromises quality of life extremely, not only have to be changed urinary canal periodically, but also get high incidence of infection and lithogenesis. Application of appendix tissue for the creation of a catheterizable stoma remains a useful technique in patients with more severe urethral injuries; ${ }^{9}$ although, the appendix is not always usable. ${ }^{10}$ The appendix may be absent or insufficient in length or quality. It may have a precarious blood supply, a short mesentery or histopathologic changes, such as chronic inflammation or fibrous lumen obstruction. ${ }^{10}$

Regarding these situations, the technique which was originally proposed by Monti et al. described a novel method using a reconfigured segment of ileum to create a catheterizable ileovesicostomy in patients. ${ }^{11}$ We just report our early experience and results with the Monti channel regarding this surgical technique in eligible patients.

\section{Materials and Methods}

From April to November 2010, three women received continent cutaneous urinary diversion using as efferent catheterizable conduit transversely tubularized ileal segments with direct implantation into the bladder wall without antireflux technique at our institution. The three patient age was 20, 41, and 43 years. All patients had a previous history of urethral distraction defect, two motor vehicle accidents, and one pelvic crush injury. One of the car
Correspondence: Dr. Hong Shen, Department of Urology, West China Hospital of Sichuan University, 37 Guoxue Alley, Chengdu, Sichuan, China.

Tel. +86.13908219730

E-mail: zhcandjay@126.com

Key words: Monti, female urethral injuries, urinary diversion.

Received for publication: 25 May 2011. Accepted for publication: 11 October 2011.

This work is licensed under a Creative Commons Attribution NonCommercial 3.0 License (CC BYNC 3.0).

(C) Copyright H. Zhang et al., 2011

Licensee PAGEPress, Italy

Urogynaecologia 2011; 25:e16

doi:10.4081/uij.2011.e16

accident injuries had also accompanied coleostenosis, vesicovaginal fistula, and urethrovaginal fistula. The others were both carried out urethral closure surgery at the local hospital when they got injuries. After pelvic fracture surgery, two of three were performed suprapubic tube, and the other was indwelled urinary canal. Informed consents were signed by all enrolled patients.

\section{Technique for Monti ileovesicostomy}

A 2-3 cm segment of bowel (usually ileum) is isolated on its mesentery in the standard fashion. If simultaneous augmentation or neobladder creation is being performed, the Monti segment should be harvested from an adjacent section, eliminating the need for an additional bowel anastomosis (Figure 1A). The segment is then opened longitudinally along the antimesenteric border and re-tubularized transversely in 2 layers with absorbable suture, over a $14 \mathrm{~F}$ catheter (Figure 1 B,C). Stay sutures placed on both ends of the tube facilitate closure by maintaining tension. The central portion is closed with running suture, and the ends are closed with interrupted suture so that adjustments to the length of the channel at the site of skin or bladder implantation can be made without disrupting the suture line. An added benefit of the Monti procedure is the ability to change the position of the mesentery along the channel by simply changing the location of the initial longitudinal incision (Figure 2 ). For instance, an asymmetrically positioned mesentery is helpful in patients with a large amount of subcutaneous tissue because more channel length is required to reach the skin than is required to reach the bladder lumen. ${ }^{12}$ 


\section{Results}

Three patients were included in this study. Causes of urethral injuries and pelvic fracture consisted of 2 motor vehicle accidents, and 1 pelvic crush injury. The time interval between injury and Monti procedure was 4, 5, and 204 months. Patients' general data, previous operative procedures and outcomes are listed in Table 1. Sonographic assessment of upper urinary tract did not reveal any pathologic findings, and mean serum creatinine level was $47.1 \mathrm{umol} / \mathrm{L}$ pre-operatively. Three patients underwent single tube technique. Mean surgical time was about $2.5 \mathrm{~h}$. Average estimated blood loss was around 350 cc. There was no need for blood transfusion or adjacent organ damage. All patients were discharged 5-6 days post-operatively. Follow-up was 5 to 12 months respectively, by outpatient and phone. Immediate post-operative complications such as hematoma and wound infection were not detected. All patients performed $14 \mathrm{~F}$ catheterization through the conduit without difficulty every 3-4 h. Mild stomal incontinence occurred in one patient at maximal bladder capacity. None of them had dehiscence, necrosis, stenosis or perforation of the tube during the followup period. Equally, there was no significant difference between pre-operative and post-operative serum creatinine levels and upper tract sonographic data.

\section{Discussion}

A variety of techniques have been used to handle problems posed by pelvic trauma associated urethral injuries in women. Owing to the physiological construction features of urethra muliebris, surgical repair of female patients with post-traumatic complex posterior urethral defects remains one of the most challenging problems in urology. In the last decade, buccal mucosa urethroplasty has increased in popularity because of its feasibility, good functional outcome, and low morbidity at the reconstructed urethra. However, treatment of long, and complicated urethral defects by buccal mucosal graft may not be useful, because of limited material. ${ }^{5}$ Other options such as bladder neck closure and suprapubic tube just could be used as a salvage procedure in such situations. But they also have some complications especially in long-term such as infection, difficulty in changing of catheter, and risk of malignancy. ${ }^{11}$ Application of the Mitrofanoff principle for urinary diversion has been revolutionized since the introduction of the continent catheterizable channel. In 1980 , Mitrofanoff first described the use of the appendix as a continent urinary stoma. However, the appendix is not always available, owing to previous appendectomy or luminal narrowing from scarring. ${ }^{5}$ Subsequently, multiple conduits have been constructed, ranging from fallopian tubes and stomach to tapered ileum. In 1997, Monti described a catheterizable channel by transverse retubularizing a short segment of bowel in order to empty their bladder. The new ileovesicostomy technique is a novel idea and provides an excellent second line option for creation of a Mitrofanoff channel. ${ }^{11}$ Only 2 to $3 \mathrm{~cm}$ segment of the ileum will usually result in a tube of $6-7 \mathrm{~cm}$ in length, when re-tubularized transversely. The caliber of such a tube allows catheterization with a $14 \mathrm{~F}$ to $16 \mathrm{~F}$ catheter, and the mucosal folds of the ileum are aligned with its longitudinal axis. These tubes have an abundant supply of blood and are able to be used anywhere inside the abdomen. If a longer channel is needed, two consecutive segments can be cut, and anastomosed together to form a tube twice as long but with mesentery only in the central portion of the tube. ${ }^{5}$ In case a larger capacity of bladder is needed, additional procedures perform at the time of the Monti procedure include bladder augmentation. A bladder augmentation will also be necessary to provide an adequate capacity, low-pressure storage reservoir. Simultaneously, maybe complications relating to catheterizable segments pertain to continence, stenosis, and ability to catheterize, with more significant morbidity relating to the bladder augmentation.

Improving health-related quality of life (HRQL) is one of the best priorities provided by Monti procedure. It is known that all
A

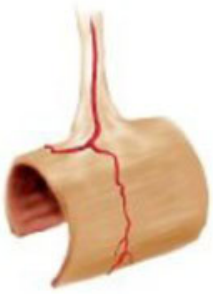

B

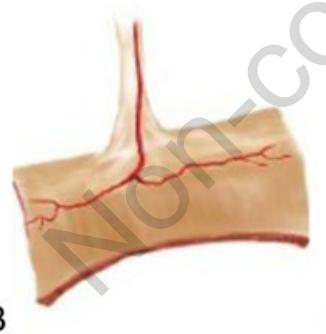

C

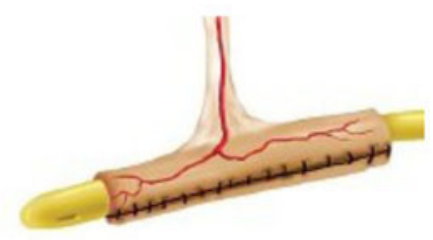

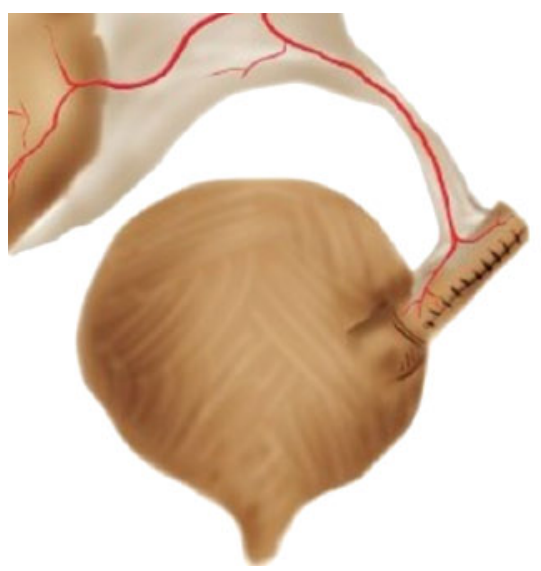

Figure 1. A) a $2-3 \mathrm{~cm}$ segment of ileum is isolated with a well-vascularized mesentery; B) transverse orientation of ileum; C) double-layer closure with running absorbable suture over a $14 \mathrm{~F}$ catheter. 5

Figure 2. The Monti tube is implanted into the bladder. ${ }^{5}$

Table 1. Patient and surgical information regarding patients undergoing Monti ileovesicostomy.

\begin{tabular}{|c|c|c|c|c|c|c|c|}
\hline Number & Diagnosis & Age (y) & $\begin{array}{l}\text { Additional } \\
\text { type of surgery }\end{array}$ & $\begin{array}{l}\text { Catheter } \\
\text { removal (W) }\end{array}$ & $\begin{array}{l}\text { Difficulty of } \\
\text { catheterization }\end{array}$ & Stomal & $\begin{array}{l}\text { Urinary } \\
\text { continence }\end{array}$ \\
\hline 1 & $\begin{array}{l}\text { Urethrovaginal fistula } \\
\text { Vesicovaginal fistula }\end{array}$ & 20 & Colpoplasty & 4 & Ease & None & $100 \%$ \\
\hline 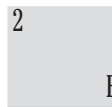 & $\begin{array}{c}\text { Pelvic trauma } \\
\text { Suprapubic tube } \\
\text { Bladder neck closure }\end{array}$ & 41 & None & 3 & Ease & None & $100 \%$ \\
\hline 3 & $\begin{array}{l}\text { Suprapubic tube } \\
\text { Ankylurethria }\end{array}$ & 43 & TVT & 3 & Ease & Bone & $100 \%$ \\
\hline
\end{tabular}

W, weeks; TVT, tension-free vaginal tape. 
patients with pelvic trauma related urethral injuries may often suffer from voiding symptoms (sensations of residual urine, interrupted or weak urine flow, difficulty of voiding and retention). Even if these patients had been managed by anastomotic repair or other operations, many complications also would accompany to these techniques. Some led to recurrent stricture, simultaneously, and need dilation of urethra in long-term seasonally. Whatever the suprapubic tube or urinary canal both bring great inconvenience to their lives. Monti procedure can commendably settle these problems as mentioned above, and allow the patients to obtain great benefits.

Another feature is urinary continence. In the three patients with follow-up periods, continence maintenance is $100 \%$ and shows no considerable changes with time. Narayanaswamy et al. reported their results with 94 Mitrofanoff procedures, of which 25 were Monti channels. Overall 23 of 25 patients were successfully catheterized at the time of the report and only 3 of 25 had stomal leakage. ${ }^{13}$ In another large series Castellan et al. reported a comparison among different types of channels for urinary and fecal incontinence, including 45 Monti urinary channels, with a mean follow-up of 38 months. Four of these channels were double Monti channels, while the others were single Monti channels. Channel replacement was performed in three patients (7\%) due to complete fibrosis, and 3 cases (7\%) had stomal

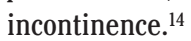

During the process of operation, we also did not use the Mitrofanoff principle to create an anti-incontinent submucosal tunnel. Yang evaluated the pressure profile of the channel tube, and detected two high-pressure zones: one in the sub-mucosal tunnel and the other at the point at which the muscle layer of the abdominal wall is crossed. These data suggest that the muscle layer of the abdominal wall is a major factor in preserving of continence. ${ }^{15}$ Our study also shows that Monti's procedure, even without the use of the Mitrofanoff principle, is a reliable technique with low incontinence and stricture rate. It can be performed in patients with an unreconstructable urethral disease, an unreconstructable bladder (e.g. exstrophy), a low leak-point pressure and neu- rogenic bladder or the inability to catheterize the urethra in a neurogenic bladder that cannot be corrected with the urethroplastic techniques, and who also do not have a suitable appendix for appendix diversion techniques. ${ }^{5}$

During the monitoring process of our outpatient and phone follow-up, after Monti's procedure shortly two of three patients ever occurred cystospasm, and led to incontinence. Our initial attempts to control urinary incontinence involve increasing clean intermittent catheterization (CIC) and taking anticholinergic agents. Gradually, the two patients' syndrome was under control. Indeed, the formation of an aesthetically desirable urinary diversion through a continent bladder stoma requires a long-term commitment by both patient and urologist to monitoring patient progress and addressing problems, both urological and otherwise, which arise over time. Therefore, regularly follow-up and timely information feedback is necessary.

\section{Conclusions}

Based on our data, the Monti channel, which the new ileovesicostomy technique originally described by Monti, is a brilliant idea and provides an excellent option for creation of a Mitrofanoff channel. It is simple to construct, provides reliable continence $(100 \%$ in our series), uses minimal amounts of small bowel. The channel transitioned well from the pediatric to the adult reconstructive setting, making it a promising alternative channel. However, evaluation of patient's satisfaction and the choice of eligible cases need more investigations with larger number of patients by longterm follow up.

\section{References}

1. Perry M0, Husmann DA. Urethral injuries in female subjects following pelvic fractures. J Urol 1992;147:139-143.

2. Hosseini J, Tavakkoli Tabassi K. Surgical repair of posterior urethral defects: review of literature and presentation of experiences. Urol J. 2008;5:215-22.

3. Cass AS, Godec CJ. Urethral injury due to external trauma. Urology 1978;11:607-11.

4. Andrich DE, Mundy AR. What is the best technique for urethroplasty? Eur Urol 2008;54:1031-41.

5. Hosseini J, Kaviani A, Mazloomfard MM Golshan AR. Monti's procedure as an alternative technique in complex urethral distraction defect. Int Braz J Urol 2010;36: 317-26.

6. Barbagli G, Lazzeri M. Surgical treatment of anterior urethral stricture diseases: brief overview. Int Braz J Urol. 2007;33: 461-9.

7. Scorer CG. The suprapubic catheter; a method of treating urinary retention. Lancet 1953;265:1222-5.

8. Barbagli G, De Angelis M, Romano G, Lazzeri M. Clinical outcome and quality of life assessment in patients treated with perineal urethrostomy for anterior urethral stricture disease. J Urol 2009;182: 548-57.

9. Freitas Filho LG, Carnevale J, Melo Filho $\mathrm{AR}$, et al. Posterior urethral injuries and the Mitrofanoff principle in children. BJU Int 2003;91:402-5.

10. Monti PR, de Carvalho JR, Arap S. The Monti procedure: applications and complications. Urology 2000;55:616-21.

11. Monti PR, Lara RC, Dutra MA, de Carvalho JR. New techniques for construction of efferent conduits based on the Mitrofanoff principle. Urology 1997;49:112-5.

12. Leslie JA, Dussinger AM, Meldrum KK. Creation of continence mechanisms (Mitrofanoff) without appendix: the Monti and spiral Monti procedures. Urol Oncol 2007;25:148-53.

13. Narayanaswamy B, Wilcox DT, Cuckow PM, et al. The Yang-Monti ileovesicostomy: a problematic channel? BJU Int 2001; 87:861-5.

14. Castellan MA, Gosalbez R Jr, Labbie A, Monti PR. Clinical applications of the Monti procedure as a continent catheterizable stoma. Urology 1999;54:152-6.

15. Yang WH. Yang needle tunneling technique in creating antireflux and continent mechanisms. J Urol 1993;150:830-4. 